\title{
Physiologically Based Pharmacokinetic Modeling of CFTR Modulation in People with Cystic Fibrosis Transitioning from Mono or Dual Regimens to Triple- Combination Elexacaftor/Tezacaftor/Ivacaftor
}

\author{
Alice Tsai (D) - Shu-Pei Wu • Eric Haseltine - Sanjeev Kumar • \\ Samuel M. Moskowitz Paul Panorchan · Kushal Shah
}

Received: June 18, 2020 / Published online: July 30, 2020

(C) The Author(s) 2020, corrected publication 2020

\section{ABSTRACT}

Introduction: The triple-combination (TC) cystic fibrosis transmembrane conductance regulator (CFTR) modulator regimen elexacaftor, tezacaftor, and ivacaftor was shown to be safe and efficacious in phase 3 trials of people with cystic fibrosis (pwCF) $\geq 12$ years of age with $\geq 1$ F508del-CFTR allele. Here, a simulation study predicted ivacaftor, tezacaftor, and elexacaftor exposures and impacts on CFTR modulation following transition from ivacaftor [a cytochrome P450 3A (CYP3A) substrate], lumacaftor (a CYP3A inducer)/ivacaftor, or tezacaftor/ivacaftor to TC.

Methods: Physiologically based pharmacokinetic (PBPK) modeling was used to evaluate plasma exposures during transition from monoor dual-combination CFTR modulator regimens to TC. PBPK models were parameterized using data from human hepatocytes to account for

Digital Features To view digital features for this article go to https://doi.org/10.6084/m9.figshare.12666467.

Electronic supplementary material The online version of this article (https://doi.org/10.1007/s41030020-00124-7) contains supplementary material, which is available to authorized users.

A. Tsai $(\bowtie) \cdot$ S.-P. Wu · E. Haseltine - S. Kumar ·

S. M. Moskowitz - P. Panorchan · K. Shah

Vertex Pharmaceuticals Incorporated, Boston, MA,

USA

e-mail: alice_tsai@vrtx.com
CYP3A induction by lumacaftor and validated to match clinical data from healthy volunteers and pwCF. Using dosing regimens for pwCF $\geq 12$ years of age, simulations were performed for ivacaftor, lumacaftor/ivacaftor, and tezacaftor/ivacaftor dosing for 14 days followed by immediate transition to elexacaftor/tezacaftor/ivacaftor dosing for 14 days. Drug exposures during transitions were compared with respective half-maximal effective concentrations $\left(\mathrm{EC}_{50}\right)$ estimated from efficacy endpoint data from clinical studies.

Results: In simulations of immediate transition from ivacaftor or tezacaftor/ivacaftor to TC, the preceding treatment had no impact on ivacaftor, tezacaftor, or elexacaftor exposures. In simulations of immediate transition from lumacaftor/ivacaftor to TC, ivacaftor exposure decreased to $64 \%$ of maximum effective concentration (EC), due to reduction in ivacaftor dose and residual CYP3A4 induction, then returned to $90-95 \%$ of maximum EC. Lumacaftor-mediated CYP3A induction resolved within approximately 2 weeks. In all simulations, ivacaftor, tezacaftor, and elexacaftor exposures approached steady state within 2 weeks following transition and, at all times, ivacaftor and $\geq 1$ CFTR corrector remained above $\mathrm{EC}_{50}$.

Conclusion: PBPK modeling indicates that immediate transition to the elexacaftor/tezacaftor/ivacaftor regimen from an ivacaftor, lumacaftor/ivacaftor, or tezacaftor/ivacaftor 
regimen results in sustained CFTR modulation in $\mathrm{pwCF} \geq 12$ years of age.

Keywords: CFTR modulator; Cystic fibrosis; Elexacaftor; Ivacaftor; Lumacaftor; Physiologically based pharmacokinetic modeling; Tezacaftor; Transition modeling; Triple combination

\section{Key Summary Points}

Why carry out this study?

Some people with cystic fibrosis (pwCF) currently receiving cystic fibrosis transmembrane conductance regulator (CFTR) modulator regimens of ivacaftor, lumacaftor/ivacaftor, or tezacaftor/ ivacaftor are transitioning to the triplecombination (TC) regimen of elexacaftor/ tezacaftor/ivacaftor.

The impact of these transitions on CFTR modulator exposures, and whether adequate exposures to achieve clinical efficacy are maintained during transition, have not been directly addressed in clinical trials.

We used physiologically based pharmacokinetic (PBPK) modeling to evaluate whether CFTR modulation is sustained during the transition from ivacaftor, lumacaftor/ivacaftor, or tezacaftor/ivacaftor to the TC regimen; this study tested the hypotheses that (1) lumacaftor induction of cytochrome P450 3A (CYP3A) would resolve within 14 days after transitioning from lumacaftor/ ivacaftor to TC and (2) that during all three transitions, the exposure of each CFTR modulator with ongoing or newly initiated dosing would stay above its halfmaximal effective concentration $\left(\mathrm{EC}_{50}\right)$ value during the transition.

\section{What was learned from the study?}

Lumacaftor-mediated CYP3A induction resolved within approximately 2 weeks; in all simulations, ivacaftor, tezacaftor, and elexacaftor exposures approached steady state within 2 weeks following transition and, at all times, ivacaftor and $\geq 1$ CFTR corrector remained above $\mathrm{EC}_{50}$.

PBPK modeling indicates that immediate transition to the elexacaftor/tezacaftor/ ivacaftor regimen from an ivacaftor, lumacaftor/ivacaftor, or tezacaftor/ ivacaftor regimen results in sustained CFTR modulation in $\mathrm{pwCF} \geq 12$ years of age.

\section{INTRODUCTION}

Cystic fibrosis (CF) is a life-shortening, multisystem disease caused by mutations in the $\mathrm{CF}$ transmembrane conductance regulator (CFTR) gene that lead to reduced quantity or function of the CFTR protein [1]. Small-molecule CFTR modulators include correctors (e.g., lumacaftor, tezacaftor, and elexacaftor) that improve CFTR processing and trafficking [2-4] and potentiators (e.g., ivacaftor) that increase CFTR channelopen probability [5]. In studies of participants with $\mathrm{CF}$, clinical benefit was observed with ivacaftor in those 6 months of age and older with CFTR gating mutations [6-10] and in those 12 years of age and older heterozygous for the F508del-CFTR mutation and a residual function CFTR mutation (F/RF) [11]. Clinical benefit was observed with lumacaftor/ivacaftor in studies of participants with CF 2 years of age and older homozygous for the F508del-CFTR mutation ( F/ $F$ genotype) [12-14]. Tezacaftor/ivacaftor also showed clinical benefit in studies of participants 6 years of age and older with $F / F$ or $F /$ RF genotypes $[11,15,16]$. 
The triple-combination (TC) regimen of elexacaftor, tezacaftor, and ivacaftor was shown to be highly efficacious in clinical studies of participants 12 years of age and older who were heterozygous for the F508del-CFTR mutation and a minimal function CFTR mutation $(F / M F)$ [17] or who had the $F / F$ genotype [18]. In participants with the $F / F$ genotype, this TC regimen showed superior efficacy across all endpoints over tezacaftor/ivacaftor [18]. Results from the study of participants with $F / M F$ genotypes showed that one copy of the F508delCFTR allele is sufficient for TC to show strong efficacy [17]. Elexacaftor/tezacaftor/ivacaftor was approved by the US Food and Drug Administration in October 2019 to treat people with CF (pwCF) 12 years of age and older with $\geq 1$ copy of the F508del-CFTR mutation [19].

Some pwCF currently receiving ivacaftor, lumacaftor/ivacaftor, or tezacaftor/ivacaftor are transitioning to this TC regimen. However, the impact of these transitions on CFTR modulator exposures, and whether adequate exposures to achieve clinical efficacy are maintained during transition, have not been directly addressed in clinical trials. Transition from lumacaftor/ivacaftor is of particular interest, as lumacaftor is a strong cytochrome P450 3A (CYP3A) inducer [20] and may lead to reduced exposures of drugs that are CYP3A substrates. Ivacaftor, tezacaftor, and elexacaftor are CYP3A substrates, with ivacaftor being a particularly sensitive CYP3A substrate $[19,21,22]$. Here, we used modeling and simulation to evaluate whether CFTR modulation is sustained during the transition period. This approach required integrating results from multiple models. First, qualified physiologically based pharmacokinetic (PBPK) models were used to simulate ivacaftor, lumacaftor, tezacaftor, and elexacaftor exposures during immediate transitions between these treatment regimens. Exposures during the transition periods were then compared to halfmaximal effective concentration $\left(\mathrm{EC}_{50}\right)$ values, as estimated from efficacy endpoint data (sweat chloride or percent predicted forced expiratory volume in $1 \mathrm{~s}$ ) from clinical studies (data on file, unpublished) to determine impacts on CFTR modulation. This study tested the hypotheses that lumacaftor induction of CYP3A would resolve within 14 days after transitioning and that the exposure of each CFTR modulator with ongoing or newly initiated dosing would stay above its $\mathrm{EC}_{50}$ value during the transition.

\section{METHODS}

\section{Ivacaftor PBPK Model}

The physiochemical properties of ivacaftor [e.g., permeability, blood-to-plasma ratio, plasma protein binding, logarithm of acid dissociation constant $\left(\mathrm{p} K_{\mathrm{a}}\right)$ ] were obtained from internal sources. All ivacaftor PBPK model parameters are available in Supplementary Table S1. The PBPK base model for oral absorption of ivacaftor was developed using an advanced dissolution, absorption, and metabolism model (ADAM) in Simcyp ${ }^{\mathrm{TM}}$ version 16 (Certara). The absorption rate constant $\left(k_{\mathrm{a}}\right)$ was predicted by the Simcyp $^{\mathrm{TM}}$ built-in simulator using Caco-2 permeability values measured in vitro and further validated to capture clinical data. For distribution, an initial steady-state volume of distribution $\left(V_{\mathrm{ss}}\right)$ of $1.89 \mathrm{~L} / \mathrm{kg}$ was estimated by allometric scaling from three species (mouse, rat, and dog) and later optimized to $1.74 \mathrm{~L} / \mathrm{kg}$. Using this initial value, the $V_{\mathrm{ss}}$, single adjusted compartment volume of distribution $\left(V_{\text {sac }}\right)$ and blood flow (SAC Q) were estimated by fitting clinical study data. Because ivacaftor is a sensitive substrate of CYP3A, and only approximately $3 \%$ parent ivacaftor was found in fecal samples in an in vivo human absorption, distribution, metabolism, and excretion (ADME) study, a fraction metabolism $\left(F_{\mathrm{m}}\right)$ value of greater than 95\% was assigned to CYP3A. A bottom-up approach was used to model elimination of ivacaftor in Simcyp ${ }^{\mathrm{TM}}$. Mechanistic human liver microsome (HLM) kinetics data [maximum rate $\left(V_{\max }\right)$; Michaelis-Menten constant $\left(K_{\mathrm{m}}\right)$ ] were estimated and further optimized to capture the clinical oral pharmacokinetic (PK) profile. Drug interaction parameters $K_{\mathrm{i}}$ (inhibitory constant) and $\mathrm{fu}_{\text {mic }}$ (fraction unbound in microsome) for CYP3A inhibition were obtained from in vitro 
experiments. The base model was validated against phase 1 clinical data (data on file, unpublished).

\section{Lumacaftor PBPK Model}

The physiochemical properties of lumacaftor (e.g., permeability, blood-to-plasma ratio, plasma protein binding, $\mathrm{p} K_{\mathrm{a}}$ ) were obtained from internal sources. All lumacaftor PBPK model parameters are available in Supplementary Table S2. First-order absorption was used to model oral absorption of lumacaftor. Absorption parameters [fraction absorbed $\left(F_{\mathrm{a}}\right), k_{\mathrm{a}}$, and lag time $\left.\left(T_{\text {lag }}\right)\right]$ were optimized based on clinical PK data. For distribution, $V_{\mathrm{ss}}, V_{\mathrm{sac}}$, and SAC $Q$ were estimated by fitting clinical trial data. Lumacaftor is not extensively metabolized, and the majority is excreted unchanged in feces [20]. Intrinsic clearance $\left(\mathrm{CL}_{\mathrm{int}}\right)$, additional hepatic clearance (HLM $\left.\mathrm{CL}_{\text {int }}\right)$, and additional systemic clearance were captured in the PBPK model using a built-in retrograde calculator. Lumacaftor is an inducer of CYP3A. Enzyme induction parameters used in Simcyp ${ }^{\mathrm{TM}}$ [maximum fold change in CYP3A4 mRNA ( $\left.\operatorname{Ind}_{\text {max }}\right)$ and concentration at half-maximum induction $\left.\left(\operatorname{Ind}_{\mathrm{C} 50}\right)\right]$ were obtained from in vitro experiments. PBPK models were parameterized using data from human hepatocytes to account for CYP3A induction by lumacaftor. The base model was validated against phase 1 clinical data (data on file, unpublished).

\section{Tezacaftor PBPK Model}

The physiochemical properties of tezacaftor (e.g., permeability, blood-to-plasma ratio, plasma protein binding, $\mathrm{p} k_{\mathrm{a}}$ ) were obtained from internal sources. Tezacaftor PBPK model parameters are available in Supplementary Table S3. The tezacaftor absorption model was developed similarly to the lumacaftor absorption model. $F_{\mathrm{a}}$ and $k_{\mathrm{a}}$ were estimated based on clinical PK data for tezacaftor. For distribution, a minimal PBPK model was used, and $V_{\text {ss }}, V_{\text {sac }}$, and SAC $Q$ were estimated by fitting clinical study data. The $F_{\mathrm{m}}$ by CYP3A for tezacaftor was estimated to be $73.2 \%$ from the human ADME study. Therefore, the CYP3A $\mathrm{CL}_{\text {int }}$ and HLM $\mathrm{CL}_{\text {int }}$ were calculated using the built-in Simcyp $^{\mathrm{TM}}$ retrograde calculator assuming that CYP3A accounts for $73.2 \%$ of the systemic oral clearance. The interaction parameters $K_{\mathrm{i}}$ and $\mathrm{fu}_{\mathrm{mic}}$ were obtained from in vitro experiments. The base model was validated against phase 1 clinical data (data on file, unpublished).

\section{Elexacaftor PBPK Model}

The physiochemical and ADME properties of elexacaftor (e.g., permeability, blood-to-plasma ratio, plasma protein binding), as well as relevant clinical data, were obtained from internal sources. Elexacaftor PBPK model parameters are available in Supplementary Table S4. First-order absorption parameters $k_{\mathrm{a}}, F_{\mathrm{a}}$, and $T_{\text {lag }}$ were estimated using clinical PK data. The drug distribution parameters of the minimal PBPK model were first estimated by optimizing three distribution parameters $\left[V_{\mathrm{ss}}, V_{\mathrm{sac}}\right.$, and intercompartmental clearance $(Q)]$ based on available clinical PK data obtained following intravenous dosing. Elexacaftor is primarily metabolized by CYP3A. The contribution of CYP3A metabolism $\left(F_{\mathrm{m}}\right)$ to the overall elimination of elexacaftor was estimated to be $67 \%$ for CYP3A; $33 \%$ of elexacaftor elimination occurs via other pathways. With this information, CYP3A CL $L_{\text {int }}$ was back-calculated in Simcyp ${ }^{\mathrm{TM}}$ using a built-in retrograde calculator. Enzyme interaction parameters $K_{\mathrm{i}}$ and $\mathrm{fu}_{\mathrm{mic}}$ were obtained from in vitro experiments. The base model was validated against phase 1 clinical data (data on file, unpublished).

\section{Simulation Design}

PK simulations were performed for the transition from ivacaftor, lumacaftor/ivacaftor, and tezacaftor/ivacaftor to the TC regimen using the previously developed and validated PBPK models for the four CFTR modulators (Supplementary Fig. 1). All simulations were performed with the default Sim-Healthy Volunteers population from the Simcyp ${ }^{\mathrm{TM}}$ virtual population library; the population was Caucasian and between 20 and 50 years of age. Information for each 
Table 1 Transition modeling simulation design for ivacaftor to elexacaftor/tezacaftor/ivacaftor

\begin{tabular}{llll}
\hline Compound type & Substrate & Inhibitor $\mathbf{1}$ & Inhibitor 2 \\
\hline Compound name & Ivacaftor & Elexacaftor & Tezacaftor \\
Route & Oral & Oral & Oral \\
Dose & $150 \mathrm{mg}$ & $200 \mathrm{mg}$ & $100 \mathrm{mg}$ \\
Time of administration & $9: 00 \mathrm{AM}$ and 9:00 PM (days 1-28) & $9: 00 \mathrm{AM}$ (days 15-28) & $9: 00 \mathrm{AM}$ (days 15-28) \\
Regimen & Once every 12 h & Once daily & Once daily \\
Fasting/fed & Fed & Fed & Fed \\
Metabolite & NA & NA & NA \\
Population & Sim-healthy volunteers & & \\
Trial number & 3 & & \\
Subject number & 3 & & \\
Gender ratio (female proportion) & 0.5 & & \\
Age (years) & $20-50$ & & \\
\hline
\end{tabular}

NA not applicable

${ }^{a}$ Simcyp ${ }^{\text {TM }}$ version 16 (Certara)

simulation is reported in Tables 1, 2, and 3. All simulations used ivacaftor, lumacaftor/ivacaftor, or tezacaftor/ivacaftor dosing regimens for pwCF 12 years of age and older for 14 days followed by the TC dosing regimen for pwCF 12 years of age and older for 14 days. PK parameters for independent PBPK modeling are reported in Supplementary Table S5. See Supplementary Methods for additional information.

\section{Software}

The PBPK analyses were performed using Simcyp $^{\mathrm{TM}}$. All plots were generated by GraphPad Prism software, version 8.1.2.

\section{Compliance with Ethics Guidelines}

This article is based on previously conducted studies and does not contain any studies with human participants or animals performed by any of the authors.

\section{RESULTS}

\section{Ivacaftor to TC Transition}

No change in the plasma drug exposure profile of ivacaftor during the transition from ivacaftor to the TC regimen was predicted (Fig. 1a); tezacaftor and elexacaftor exposures increased and reached steady state within 1 week (Fig. 1b, c).

\section{Lumacaftor/Ivacaftor to TC Transition}

Simulations showed that when transitioning from lumacaftor/ivacaftor (lumacaftor $400 \mathrm{mg}$ and ivacaftor $250 \mathrm{mg}$ once every $12 \mathrm{~h}$ ) to the TC regimen (elexacaftor $200 \mathrm{mg}$ once daily, tezacaftor $100 \mathrm{mg}$ once daily, and ivacaftor $150 \mathrm{mg}$ once every $12 \mathrm{~h}$ ), ivacaftor plasma exposure transiently decreased upon immediate transition and then gradually increased to steady state on day 14 after the transition (Fig. 2a), decreasing to a nadir of $64 \%$ of maximum effective concentration (EC) and then 
Table 2 Transition modeling simulation design for lumacaftor/ivacaftor to elexacaftor/tezacaftor/ivacaftor

\begin{tabular}{|c|c|c|c|c|c|}
\hline Compound type & Substrate & & Inhibitor 1 & Inhibitor 2 & Inhibitor 3 \\
\hline Compound name & Ivacaftor & & Lumacaftor & Elexacaftor & Tezacaftor \\
\hline Route & Oral & & Oral & Oral & Oral \\
\hline Dose & $250 \mathrm{mg}$ & $150 \mathrm{mg}$ & $400 \mathrm{mg}$ & $200 \mathrm{mg}$ & $100 \mathrm{mg}$ \\
\hline $\begin{array}{l}\text { Time of } \\
\text { administration }\end{array}$ & $\begin{array}{l}\text { 9:00 AM and 9:00 PM } \\
\quad(\text { days 1-14) }\end{array}$ & $\begin{array}{l}\text { 9:00 AM and 9:00 PM } \\
\text { (days 15-28) }\end{array}$ & $\begin{array}{c}9: 00 \text { AM } \\
\text { (days } \\
1-14)\end{array}$ & $\begin{array}{c}9: 00 \text { AM } \\
\text { (days } \\
15-28)\end{array}$ & $\begin{array}{c}\text { 9:00 AM (days } \\
15 \text { to } 28 \text { ) }\end{array}$ \\
\hline Regimen & Once every $12 \mathrm{~h}$ & & $\begin{array}{l}\text { Once every } \\
12 \mathrm{~h}\end{array}$ & Once daily & Once daily \\
\hline Fasting/fed & Fed & & Fed & Fed & Fed \\
\hline Metabolite & NA & & NA & NA & NA \\
\hline Population & Sim-healthy volunteers ${ }^{a}$ & & & & \\
\hline Trial number & 3 & & & & \\
\hline Subject number & 3 & & & & \\
\hline $\begin{array}{l}\text { Gender ratio (female } \\
\text { proportion) }\end{array}$ & 0.5 & & & & \\
\hline Age (years) & $20-50$ & & & & \\
\hline
\end{tabular}

increasing to $90-95 \%$ EC (EC $90-95)$ within approximately 3 days. The exposure of lumacaftor decreased slowly and was completely eliminated on day 8 after discontinuation of lumacaftor/ivacaftor (Fig. 2b); CYP3A induction due to lumacaftor was predicted to resolve within approximately 2 weeks. The plasma exposures of tezacaftor and elexacaftor increased over time, reaching steady state on day 14 after transition (Fig. 2c, d); these exposures were maintained above $\mathrm{EC}_{50}$ at all times. Exposures of tezacaftor and elexacaftor were not greatly impacted by lumacaftor-mediated CYP3A induction during this transition. At all times during the transition period, exposures of $\geq 1$ corrector (lumacaftor, tezacaftor, or elexacaftor) remained above their respective $\mathrm{EC}_{50}$.

\section{Tezacaftor/Ivacaftor to TC Transition}

When transitioning from tezacaftor/ivacaftor to the TC regimen, ivacaftor and tezacaftor exposures were maintained (Fig. 3a, b). The exposure of elexacaftor increased and reached steady state within 1 week (Fig. 3c).

\section{DISCUSSION}

Our PBPK models of ivacaftor, lumacaftor, tezacaftor, and elexacaftor were developed and validated in an adult healthy Caucasian volunteer population between 20 and 50 years of age. This virtual population was the most appropriate Simcyp ${ }^{\mathrm{TM}}$ population to use compared with other available virtual populations of differing races or functional renal or hepatic status 
Table 3 Transition modeling simulation design for tezacaftor/ivacaftor to elexacaftor/tezacaftor/ivacaftor

\begin{tabular}{llll}
\hline Compound type & Substrate & Inhibitor 1 & Inhibitor 2 \\
\hline Compound name & Ivacaftor & Elexacaftor & Tezacaftor \\
Route & Oral & Oral & Oral \\
Dose & $150 \mathrm{mg}$ & $200 \mathrm{mg}$ & $100 \mathrm{mg}$ \\
Time of administration & $9: 00 \mathrm{AM}$ and 9:00 PM (days 1-28) & $9: 00 \mathrm{AM}$ (days 15-28) & $9: 00 \mathrm{AM}$ (days 1-28) \\
Regimen & Once every 12 h & Once daily & Once daily \\
Fasting/fed & Fed & Fed & Fed \\
Metabolite & $\mathrm{NA}$ & $\mathrm{NA}$ & $\mathrm{NA}$ \\
Population & Sim-healthy volunteers & \\
Trial number & 3 & & \\
Subject number & 3 & & \\
Gender ratio (female proportion) & 0.5 & & \\
Age (years) & $20-50$ & & \\
\hline
\end{tabular}

NA not applicable

${ }^{a}$ Simcyp ${ }^{\mathrm{TM}}$ version 16 (Certara)

because most pwCF are white [23, 24], and PK parameters are not expected to be substantially different between healthy volunteers and pwCF. Protein expression and enzyme activity of CYP3A are expected to be similar for all people 12 years of age and older, including those with CF [25]. Moreover, the differences between simulated plasma exposures in the virtual healthy population and those observed in pwCF 12 years of age and older were negligible, and the extent of drug-drug interactions is similar between adults and adolescents [26], indicating that the developed PBPK relationship can be applied to pwCF 12 years of age and older.

Using the PBPK modeling approach, we simulated transitions from ivacaftor, lumacaftor/ivacaftor, and tezacaftor/ivacaftor to the TC regimen elexacaftor/tezacaftor/ivacaftor to evaluate whether CFTR modulation was maintained during transitions between these CFTR modulator regimens. This question is particularly germane when transitioning from lumacaftor/ivacaftor, because lumacaftor is a strong CYP3A inducer and ivacaftor is a sensitive CYP3A substrate whose exposure is impacted by lumacaftor [20]. When transitioning from lumacaftor/ivacaftor to TC, pwCF lower their ivacaftor dose from $250 \mathrm{mg}$ once every $12 \mathrm{~h}$ to $150 \mathrm{mg}$ once every $12 \mathrm{~h}$ and discontinue lumacaftor. Following this switch in therapies, lumacaftor exposure is predicted to decrease and ivacaftor exposure is predicted to increase, reaching steady state on day 14 after the transition. During the transition, tezacaftor and elexacaftor were minimally impacted and remained above their respective $\mathrm{EC}_{50}$ throughout. Based on the simulations in this study, ivacaftor exposure is predicted to remain above $\mathrm{EC}_{50}$ throughout the transition, decreasing to a nadir of $64 \%$ of maximum EC and then increasing to $\mathrm{EC}_{90-95}$ within approximately 3 days. Lumacaftor-mediated CYP3A induction is predicted to resolve within approximately 2 weeks.

Tezacaftor and elexacaftor have low potential to inhibit or induce CYP3A [19]. In all simulated transitions, no change in ivacaftor exposure due to addition of tezacaftor and/or elexacaftor was predicted. Similarly, no change in tezacaftor exposure during the transition 
(a)
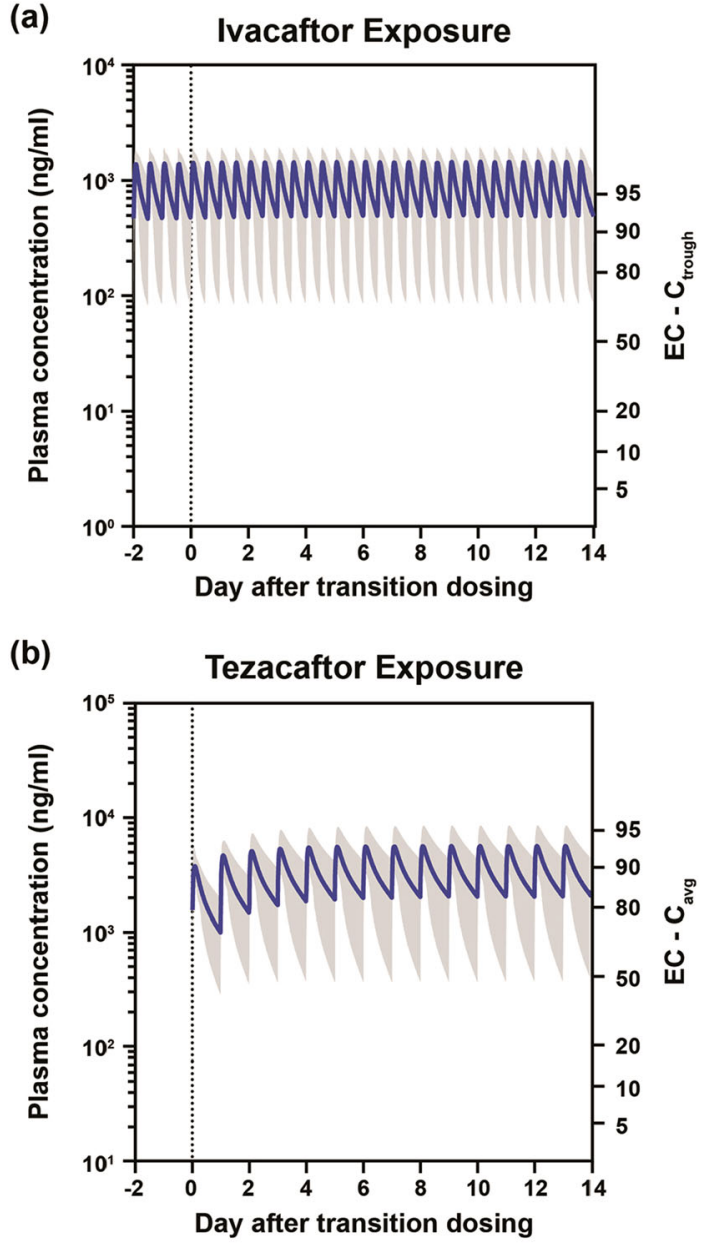

(c)

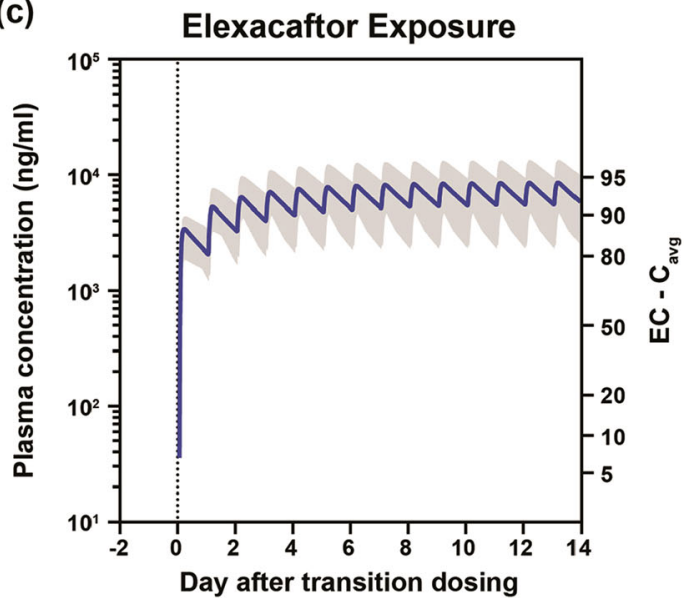

Fig. 1 Plasma exposure of CFTR modulators after transition from ivacaftor to elexacaftor/tezacaftor/ivacaftor. a Ivacaftor exposure. b Tezacaftor exposure. c Elexacaftor exposure. In all figures, shaded area indicates 5th-95th percentile. $C_{\text {avg }}$ average concentration, $C_{\text {trough }}$ trough concentration, $E C$ effective concentration from tezacaftor/ivacaftor to TC was predicted. Finally, no change in the plasma exposure profile was predicted for elexacaftor after transition in any simulation.

Each individual CFTR modulator in the TC regimen has its own target EC. In these simulations, $\mathrm{EC}_{50}$ was chosen as the threshold above which CFTR modulator exposures should remain during the transition period; however, after transitioning, all modulators reached or returned to their target EC. $\mathrm{EC}_{50}$ is commonly used when analyzing drug exposure and was used in a previous analysis of simulated transitions between lumacaftor/ivacaftor and tezacaftor/ivacaftor [27]; during those simulated transitions, the CFTR modulators remained above $\mathrm{EC}_{50}$ and CFTR modulation was sustained. Keeping exposures above $\mathrm{EC}_{50}$ is important during transitions between treatments, because the more time that is spent above $\mathrm{EC}_{50}$, the more likely it is that the drug will show efficacy.

A limitation of this study is that all the simulations were based on available clinical data from study participants 12 years of age and older. Additional simulations will be needed to address transitions in the pediatric CF population due to the impact of CYP3A enzyme ontogeny, which typically occurs between 0 and 2 years of age, as well as potential differences in pediatric dosing regimens.

\section{CONCLUSIONS}

The PBPK modeling approach is useful for integrating all available in vitro and in vivo ADME and PK data to predict the impact of an immediate transition from ivacaftor, lumacaftor/ivacaftor, or tezacaftor/ivacaftor to elexacaftor/tezacaftor/ivacaftor on the exposures of those CFTR modulators in pwCF 12 years of age and older. Given the CYP3A-inducing effects of lumacaftor, these simulations predict that during all three of these transitions to the TC regimen, exposures of ivacaftor, tezacaftor, and elexacaftor will reach steady state within 2 weeks of the transition and CFTR modulation will be sustained. 
(a)

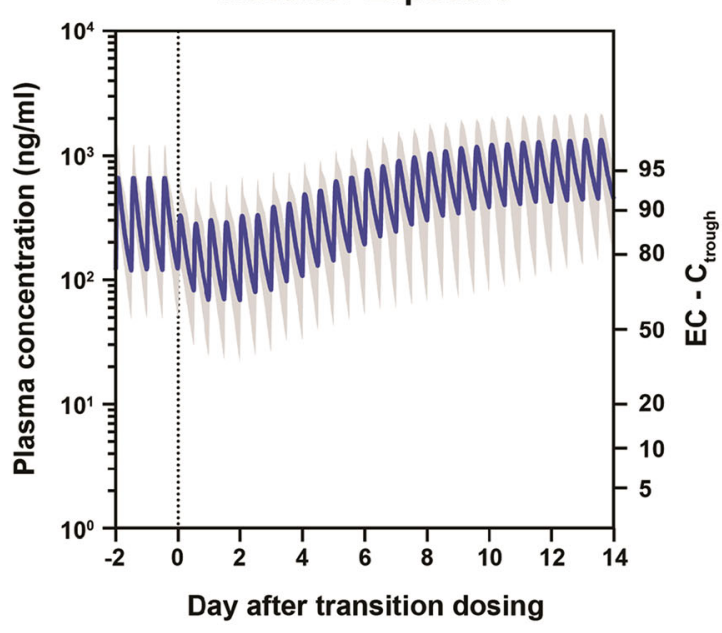

(c)

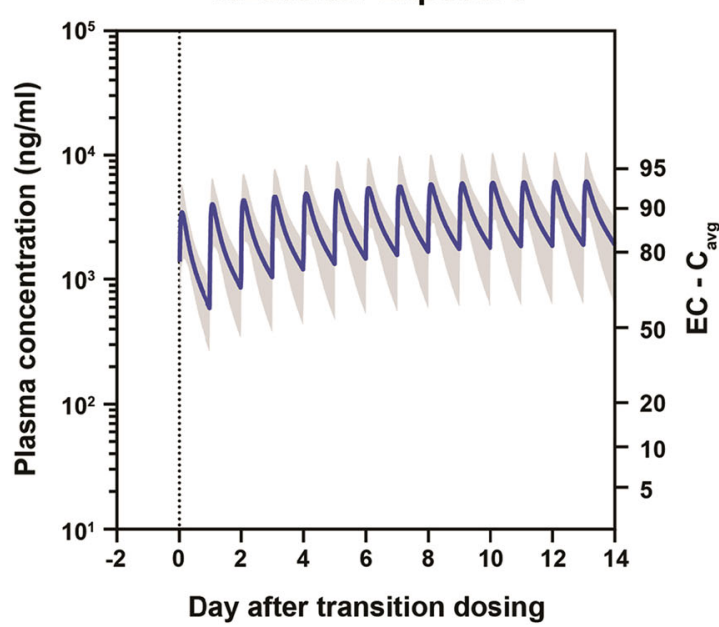

Fig. 2 Plasma exposures of CFTR modulators after transition from lumacaftor/ivacaftor to elexacaftor/tezacaftor/ivacaftor. a Ivacaftor exposure. b Lumacaftor exposure. $\mathbf{c}$ Tezacaftor exposure. $\mathbf{d}$ Elexacaftor exposure. In all

\section{ACKNOWLEDGEMENTS}

Funding. This study was funded by Vertex Pharmaceuticals Incorporated, Boston, MA, USA. The journal's Rapid Service Fee was funded by Vertex Pharmaceuticals Incorporated, Boston, MA, USA.

Medical Writing, Editorial, and Other Assistance. Editorial coordination and support
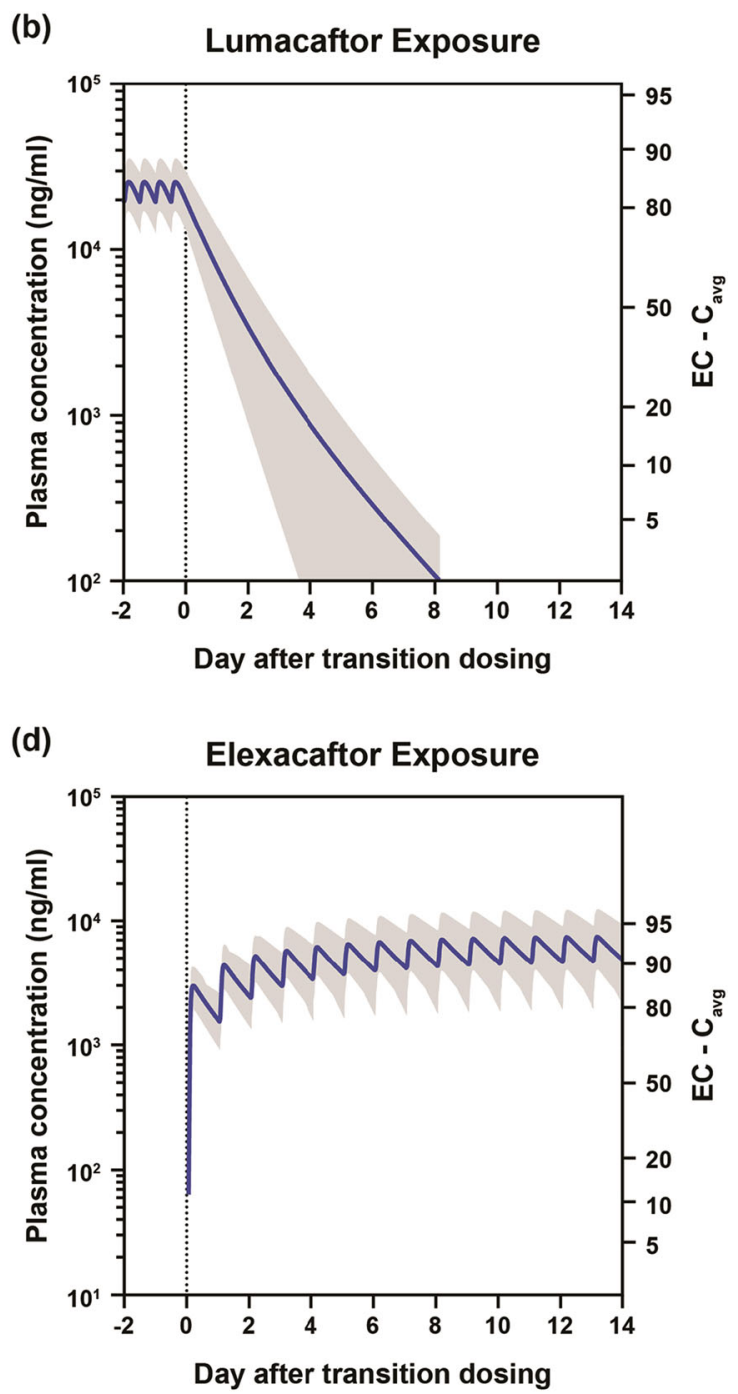

figures, shaded area indicates 5th-95th percentile. $C_{\text {avg }}$ average concentration, $C_{\text {trough }}$ trough concentration, $E C$ effective concentration

were provided by Morgan Deng, PharmD, of Vertex Pharmaceuticals Incorporated; Morgan Deng may own stock or stock options in that company. Medical writing and editorial support were provided under the direction of the authors by Christopher Edwards, PhD, and Karen Kaluza Smith, PhD, CMPP, with support from Vertex Pharmaceuticals Incorporated.

Authorship. All named authors meet the International Committee of Medical Journal 
(a)

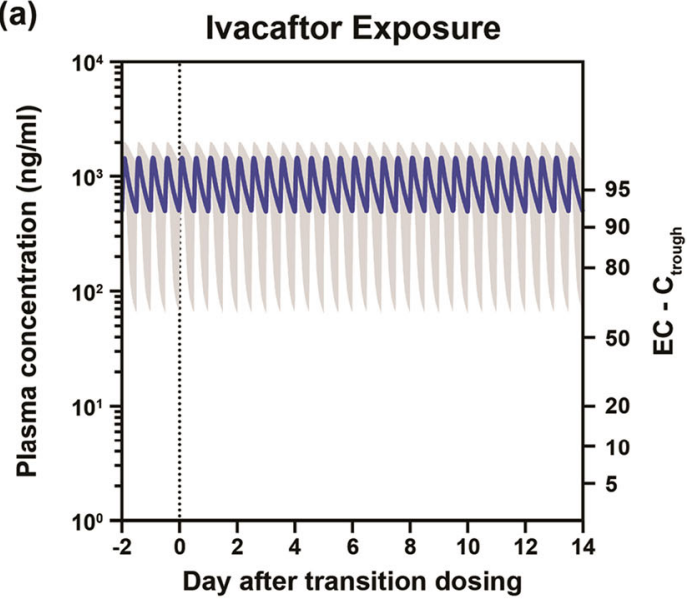

(b)

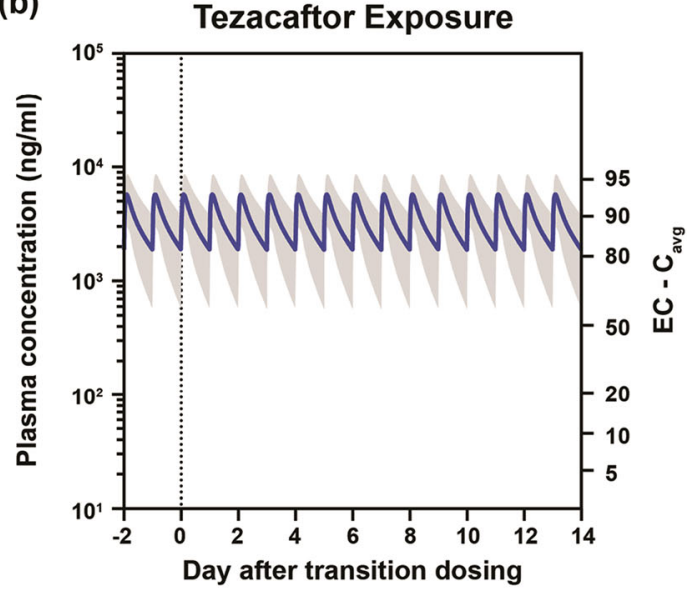

(c)

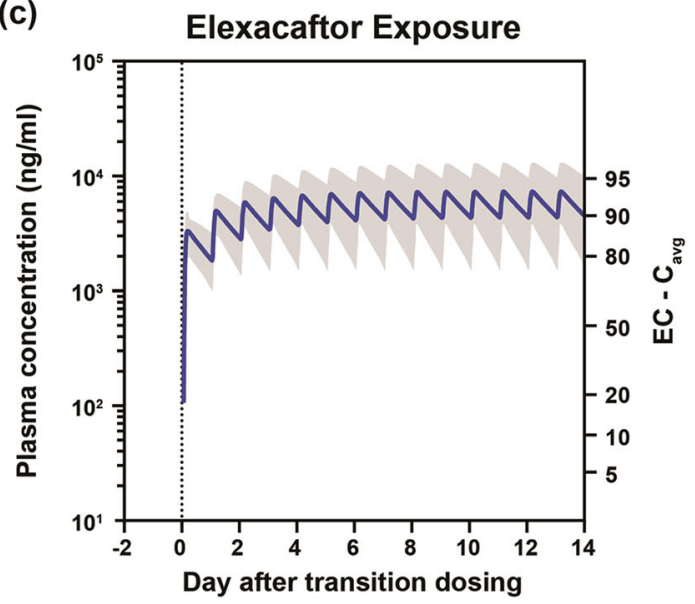

Fig. 3 Plasma exposures of CFTR modulators after transition from tezacaftor/ivacaftor to elexacaftor/tezacaftor/ivacaftor. a Ivacaftor exposure. b Tezacaftor exposure. c Elexacaftor exposure. In all figures, shaded area indicates 5th-95th percentile. $C_{\text {avg }}$ average concentration, $C_{\text {trough }}$ trough concentration, $E C$ effective concentration
Editors (ICMJE) criteria for authorship for this article, take responsibility for the integrity of the work as a whole, and have given their approval for this version to be published.

Disclosures. Alice Tsai, Shu-Pei $\mathrm{Wu}$, Eric Haseltine, Sanjeev Kumar, Samuel M. Moskowitz, Paul Panorchan, and Kushal Shah are employees of Vertex Pharmaceuticals Incorporated and may own stock or stock options in that company. Alice Tsai, Shu-Pei Wu, Eric Haseltine, Sanjeev Kumar, Samuel M. Moskowitz, Paul Panorchan, and Kushal Shah have received nonfinancial support (assistance with manuscript preparation) from ArticulateScience LLC, which received funding from Vertex Pharmaceuticals Incorporated. Eric Haseltine: Pending patent for Compositions and Methods for Treatment of Cystic Fibrosis (WO 2019/010092, PCT/US2018/040427). Samuel M. Moskowitz: Pending patent for Methods of Treatment for Cystic Fibrosis; pending patent for Methods of Treatment of Cystic Fibrosis; pending patent for Pharmaceutical Compositions for Treating Cystic Fibrosis.

Compliance with Ethics Guidelines. This article is based on previously conducted studies and does not contain any studies with human participants or animals performed by any of the authors.

Data Availability. The datasets generated during and/or analyzed during the current study are available from the corresponding author on reasonable request. Vertex Pharmaceuticals Incorporated is committed to advancing medical science and improving the health of people with cystic fibrosis. This includes the responsible sharing of clinical trial data with qualified researchers. Proposals for the use of these data will be reviewed by a scientific board. Approvals are at the discretion of Vertex Pharmaceuticals Incorporated and will be dependent on the nature of the request, the merit of the research proposed, and the intended use of the data. Please contact CTDS@vrtx.com if you would like to submit a proposal or need more information. 
Open Access. This article is licensed under a Creative Commons Attribution-NonCommercial 4.0 International License, which permits any non-commercial use, sharing, adaptation, distribution and reproduction in any medium or format, as long as you give appropriate credit to the original author(s) and the source, provide a link to the Creative Commons licence, and indicate if changes were made. The images or other third party material in this article are included in the article's Creative Commons licence, unless indicated otherwise in a credit line to the material. If material is not included in the article's Creative Commons licence and your intended use is not permitted by statutory regulation or exceeds the permitted use, you will need to obtain permission directly from the copyright holder. To view a copy of this licence, visit http://creativecommons.org/licenses/by$\mathrm{nc} / 4.0 /$.

\section{REFERENCES}

1. Elborn JS. Cystic fibrosis. Lancet. 2016;388: 2519-31.

2. Van Goor F, Hadida S, Grootenhuis PDJ, et al. Correction of the F508del-CFTR protein processing defect in vitro by the investigational drug VX-809. Proc Natl Acad Sci USA. 2011;108:18843-8.

3. Van Goor F, Grootenhuis P, Hadida S, et al. Nonclinical profile of the CFTR corrector VX-661. Pediatr Pulmonol. 2016;51 [abstract 217].

4. Keating D, Marigowda G, Burr L, et al. VX445-tezacaftor-ivacaftor in patients with cystic fibrosis and one or two Phe508del alleles. N Engl J Med. 2018;379:1612-20.

5. Van Goor F, Hadida S, Grootenhuis PD, et al. Rescue of CF airway epithelial cell function in vitro by a CFTR potentiator, VX-770. Proc Natl Acad Sci USA. 2009; 106:18825-30.

6. Davies J, Wang LT, Panorchan P, et al. Ivacaftor (IVA) treatment in patients 6 to $<12$ months old with cystic fibrosis with a CFTR gating mutation: results of a 2-part, single-arm, phase 3 study. J Cyst Fibros. 2019;18:S11 [abstract WS06-4].

7. Rosenfeld M, Wainwright CE, Higgins $\mathrm{M}$, et al. Ivacaftor treatment of cystic fibrosis in children aged 12 to less than 24 months and with a CFTR gating mutation (ARRIVAL): a phase 3 single-arm study. Lancet Respir Med. 2018;6:545-53.

8. Davies JC, Cunningham S, Harris WT, et al. Safety, pharmacokinetics, and pharmacodynamics of ivacaftor in patients aged 2-5 years with cystic fibrosis and a CFTR gating mutation (KIWI): an open-label, single-arm study. Lancet Respir Med. 2016;4: 107-15.

9. Ramsey BW, Davies J, McElvaney NG, et al. A CFTR potentiator in patients with cystic fibrosis and the G551D mutation. N Engl J Med. 2011;365: 1663-722.

10. De Boeck K, Munck A, Walker S, et al. Efficacy and safety of ivacaftor in patients with cystic fibrosis and a non-G551D gating mutation. J Cyst Fibros. 2014;13:674-80.

11. Rowe SM, Daines C, Ringshausen FC, et al. Tezacaftor-ivacaftor in residual-function heterozygotes with cystic fibrosis. N Engl J Med. 2017;377: 2024-35.

12. McNamara JJ, McColley SA, Marigowda G, et al. Safety, pharmacokinetics, and pharmacodynamics of lumacaftor and ivacaftor combination therapy in children aged 2-5 years with cystic fibrosis homozygous for F508del-CFTR: an open-label phase 3 study. Lancet Respir Med. 2019;7:325-35.

13. Ratjen F, Hug C, Marigowda G, et al. Efficacy and safety of lumacaftor and ivacaftor in patients aged 6-11 years with cystic fibrosis homozygous for F508del-CFTR: a randomised, placebo-controlled phase 3 trial. Lancet Respir Med. 2017;5:557-67.

14. Wainwright CE, Elborn JS, Ramsey BW, et al. Lumacaftor-ivacaftor in patients with cystic fibrosis homozygous for Phe508del CFTR. N Engl J Med. 2015;373:220-31.

15. Walker S, Flume P, McNamara J, et al. A phase 3 study of tezacaftor in combination with ivacaftor in children aged 6 through 11 years with cystic fibrosis. J Cyst Fibros. 2019;18:708-13.

16. Taylor-Cousar J, Munck A, McKone EF, et al. Tezacaftor-ivacaftor in patients with cystic fibrosis homozygous for Phe508del. $N$ Engl J Med. 2017;377:2013-23.

17. Middleton PG, Mall MA, Drevinek P, et al. Elexacaftor-tezacaftor-ivacaftor for cystic fibrosis with a single Phe508del allele. N Engl J Med. 2019;381: 1809-19.

18. Heijerman HGM, McKone EF, Downey DG, et al. Efficacy and safety of the elexacaftor plus tezacaftor plus ivacaftor combination regimen in people with cystic fibrosis homozygous for the F508del 
mutation: a double-blind, randomised, phase 3 trial. Lancet. 2019;394:1940-8.

19. Vertex Pharmaceuticals Incorporated. Trikafta (elexacaftor/tezacaftor/ivacaftor) [package insert]. Boston: Vertex Pharmaceuticals Incorporated; 2019.

20. Vertex Pharmaceuticals Incorporated. Orkambi (lumacaftor/ivacaftor) [package insert]. Boston: Vertex Pharmaceuticals Incorporated; 2018.

21. Vertex Pharmaceuticals Incorporated. Kalydeco (ivacaftor) [package insert]. Boston: Vertex Pharmaceuticals Incorporated; 2019.

22. Vertex Pharmaceuticals Incorporated. Symdeko (tezacaftor/ivacaftor) [package insert]. Boston: Vertex Pharmaceuticals Incorporated; 2019.

23. Cystic Fibrosis Foundation. Cystic Fibrosis Foundation Patient Registry: 2017 annual data report. https:// www.cff.org/Research/Researcher-Resources/PatientRegistry/2017-Patient-Registry-Annual-Data-Report. pdf. Accessed June 17, 2020.
24. O'Sullivan BP, Freedman SD. Cystic fibrosis. Lancet. 2009;373:1891-904.

25. Johnson TN, Rostami-Hodjegan A, Tucker GT. Prediction of the clearance of eleven drugs and associated variability in neonates, infants and children. Clin Pharmacokinet. 2006;45:931-56.

26. Salem F, Johnson TN, Barter ZE, Leeder JS, RostamiHodjegan A. Age related changes in fractional elimination pathways for drugs: assessing the impact of variable ontogeny on metabolic drugdrug interactions. J Clin Pharmacol. 2013;53: 857-65.

27. Wu S, Garg V, Tsai A, et al. Sustained CFTR correction and potentiation is predicted during transitions between lumacaftor/ivacaftor and tezacaftor/ivacaftor-based regimens. Pediatr Pulmonol. 2017;52 [abstract 253]. 